\title{
Technè
}

La science au service de l'histoire de l'art et de la préservation des biens culturels

42 | 2015

Science et conservation

\section{De La Source aux Trois baigneuses : les tableaux à transformations de Gustave Courbet}

From The Source to Three Bathers: metamorphosis of Gustave Courbet's paintings

\section{Bruno Mottin}

\section{(2) OpenEdition}

\section{Journals}

Édition électronique

URL : http://journals.openedition.org/techne/7155

DOI : 10.4000/techne.7155

ISSN : 2534-5168

Éditeur

C2RMF

\section{Édition imprimée}

Date de publication : 1 décembre 2015

Pagination : 101-102

ISBN : 978-2-7118-6249-8

ISSN : 1254-7867

\section{Référence électronique}

Bruno Mottin, «De La Source aux Trois baigneuses : les tableaux à transformations de Gustave

Courbet », Technè [En ligne], 42 | 2015, mis en ligne le 01 décembre 2015, consulté le 11 mars 2021

URL : http://journals.openedition.org/techne/7155; DOI : https://doi.org/10.4000/techne.7155

\section{(c) $(1) \&$}

La revue Technè. La science au service de l'histoire de l'art et de la préservation des biens culturels est mise à disposition selon les termes de la Licence Creative Commons Attribution - Pas d'Utilisation Commerciale - Pas de Modification 4.0 International. 


\section{Bruno Mottin \\ De La Source aux Trois baigneuses : les tableaux à transformations de Gustave Courbet}

From The Source to Three Bathers:

metamorphosis of Gustave Courbet's paintings

Résumé. L'article présente brièvement les résultats d'une étude de laboratoire conduite sur deux tableaux de Gustave Courbet. Il montre que le tableau des Trois Baigneuses était au départ une feuille d'étude, sur laquelle le peintre a travaillé à plusieurs compositions, avant de la maroufler sur toile en lui conférant ainsi le statut de peinture de chevalet.

Mots-clés. Gustave Courbet, Trois baigneuses, La Source.
Abstract. The article summarizes the results of a laboratory study carried out on two works painted by Gustave Courbet. It shows that the painting of the Three Bathers was originally a sheet of studies, on which the artist was working on several compositions. He then mounted it on canvas and it thus acquired the status of an easel painting.

Keywords. Gustave Courbet, Three Bathers, The Spring.

L'étude de laboratoire des tableaux de Gustave Courbet réserve fréquemment des surprises, car ce maître extraordinairement créatif n'a jamais hésité à transformer radicalement ses compositions au cours de leur élaboration ${ }^{1}$. L'examen des Trois baigneuses du musée du Petit Palais en est un exemple particulièrement frappant, comme nous avons tenté de le montrer dans un précédent texte dont nous présentons ici les lignes saillantes ${ }^{2}$.

L'œuvre représentant les Trois baigneuses n'a pas été conçue d'emblée comme une peinture. C'était initialement une simple feuille d'étude sur papier épais, de plus petit format que la peinture actuelle, qui a été utilisée par Courbet pour mettre au point des compositions successives. Celles-ci peuvent être observées grâce à la radiographie (fig.4 de l'article ColletSindaco), à la réflectographie infrarouge et à l'imagerie par spectrométrie de fluorescence X. Le peintre a d'abord utilisé sa feuille en la disposant en longueur pour esquisser une femme nue allongée au bord de l'eau, accoudée sur un rocher. La composition peut être datée vers 1862, par analogie avec un $N u$ au ruisseau qui a été redécouvert récemment au musée El Guézireh du Caire. Mais Courbet ne s'est pas arrêté là ; à la manière d'Ingres, qui superpose des variantes à ses compositions, le peintre a transformé la pose en redressant les bras de la jeune femme au-dessus de sa tête, pour créer une figure dont les contours sinueux sont en rapport avec les recherches de stylisation qu'il mène autour de 1868.

Vers 1868, Courbet s'intéresse également au thème des baigneuses debout, seules ou en groupe, dont l'une des versions les plus célèbres est La Source du musée d'Orsay (RF 2240, fig. 1). L'œuvre représente aujourd'hui une femme nue vue de dos se baignant dans un sous-bois, mais la radiographie

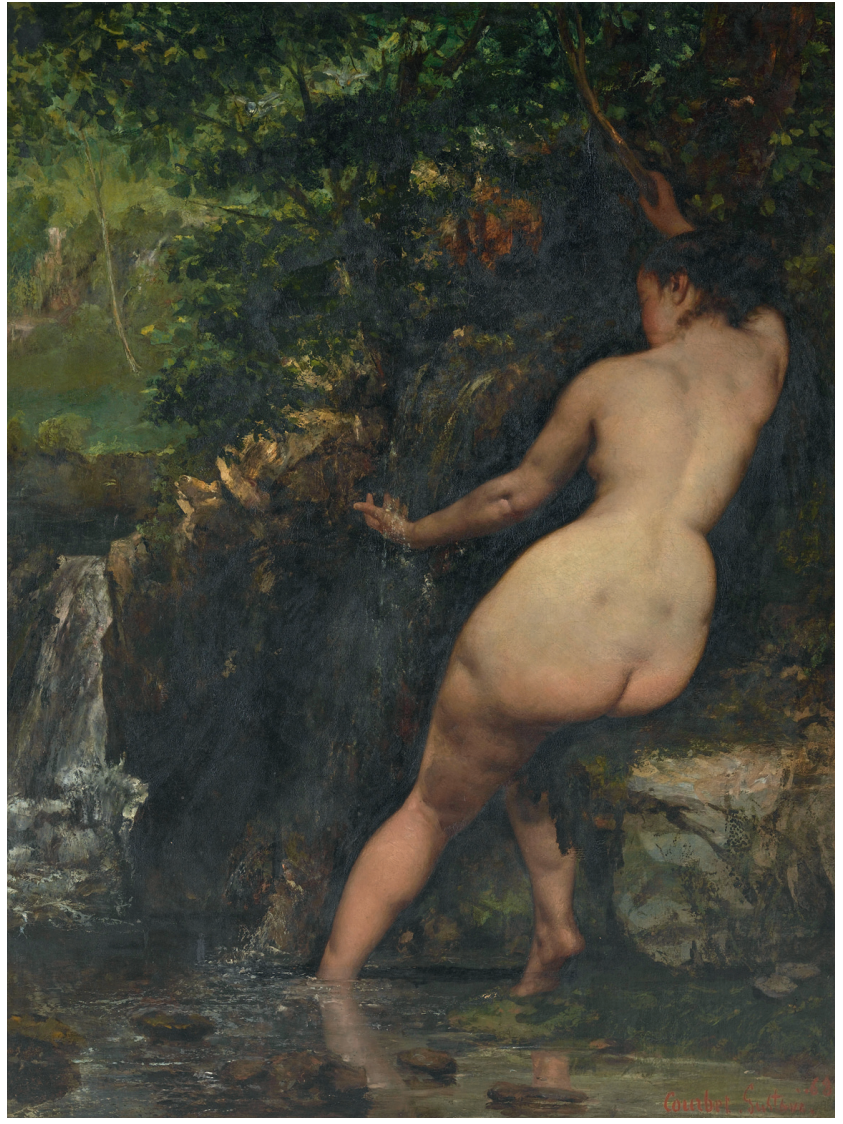

Fig. 1. Gustave Courbet, La Source, huile sur toile. Paris, musée d'Orsay. () Musée d'Orsay, Dist. RMN-Grand Palais/Patrice Schmidt. 
montre que la composition en recouvre une autre, à trois baigneuses, qui ressemble fortement à la composition du Petit Palais (fig. 2). On note que Courbet a hésité, pour le tableau aujourd'hui au musée d'Orsay, sur l'attitude du nu le plus à gauche, car son image apparaît floue sur la radiographie. Pour retravailler cette figure, le peintre est revenu à sa feuille d'étude, qu'il a basculée dans un sens vertical. Il, a modifié les proportions du nu allongé pour en faire une baigneuse glissant dans l'eau, a ajouté une femme nue vue de dos sur la droite et a glissé entre les deux jeunes femmes la tête d'un troisième personnage. Cet exemple de transformation d'une composition en une autre par basculement de 90 degrés est emblématique de la dextérité du peintre et semble unique dans l'histoire de la peinture. Pendant une brève période, les œuvres du musée d'Orsay et du Petit Palais ont donc été semblables, avant de suivre des voies divergentes. Pour $L a$ Source, Courbet a choisi de ne retenir que le personnage de droite, au modelé sculptural. En revanche, il a développé le thème des trois baigneuses sur l'œuvre du Petit Palais en faisant passer la feuille d'étude au rang de peinture de chevalet grâce à son marouflage de la feuille sur un support de toile et à son agrandissement sur quatre côtés. Ayant ainsi aéré la scène, il a pu équilibrer la composition en déplaçant le nu de droite. Il aurait probablement terminé cette œuvre en peu de jours s'il n'avait participé aux événements de la Commune et si la composition ne lui avait été volée pendant son emprisonnement. Elle a été retrouvée chez un marchand quelques années plus tard, alors que Courbet était exilé en Suisse et n'a pu la retoucher.

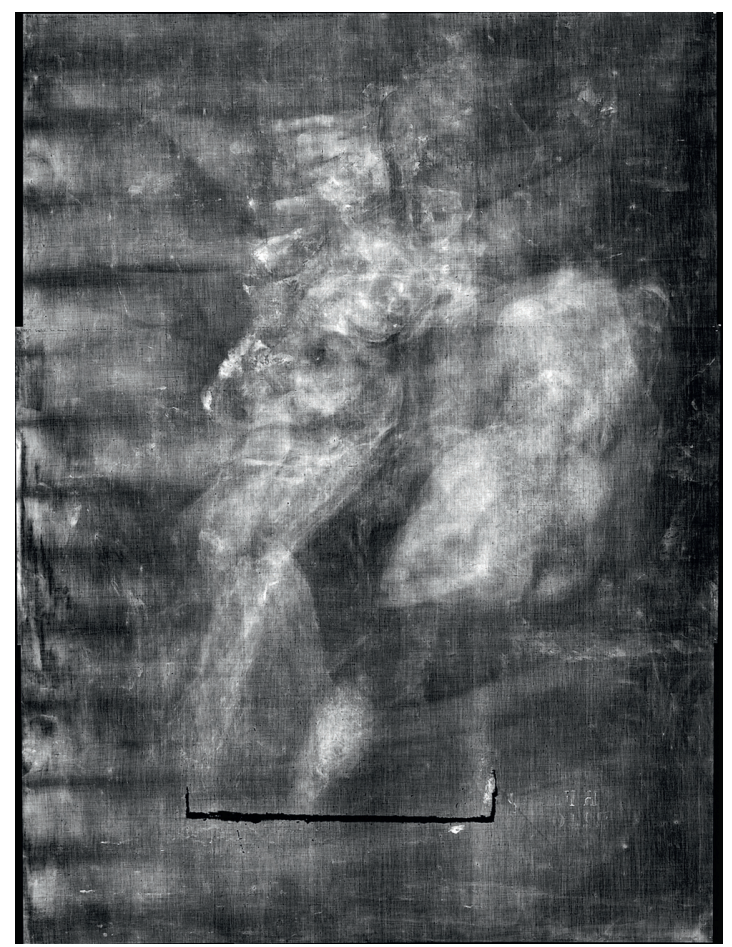

Fig. 2. Gustave Courbet, La Source, radiographie. (C) C2RMF.

\section{Notes}

1. Mottin B., 2007-2008, p. 71-80.

2. Mottin B., 2014.

\section{Bibliographie}

Mottin B., 2007-2008, « Des œuvres à la genèse complexe : Courbet sous l'œil du laboratoire ", Gustave Courbet, cat. exp. Paris, Grand Palais, New York, Metropolitan Museum, Montpellier, musée Fabre, p. 71-80.

Mottin B., 2014, "From One Picture

to Another: Les 'Trois Baigneuses'”,

Gustave Courbet, cat. exp. Bale,

Fondation Beyeler, 2014, p. 106-112. 